\title{
Cholangiocarcinoma Evaluation via Imaging and Artificial Intelligence
}

\author{
Chun Mei Yang Jian Shu \\ Department of Radiology, The Affiliated Hospital of Southwest Medical University, Luzhou, PR China
}

\section{Keywords}

Cholangiocarcinoma $\cdot$ Imaging evaluation $\cdot$ Artificial intelligence

\section{Abstract}

Background: Cholangiocarcinoma (CCA) is a relatively rare malignant biliary system tumor, and yet it represents the second most common primary hepatic neoplasm, following hepatocellular carcinoma. Regardless of the type, location, or etiology, the survival prognosis of these tumors remains poor. The only method of cure for CCA is complete surgical resection, but part of patients with complete resection are still subject to local recurrence or distant metastasis. Summary: Over the last several decades, our understanding of the molecular biology of CCA has increased tremendously, diagnostic and evaluative techniques have evolved, and novel therapeutic approaches have been established. Key Messages: This review provides an overview of preoperative imaging evaluations of CCA. Furthermore, relevant information about artificial intelligence (Al) in medical imaging is discussed, as well as the development of Al in CCA treatment.

(c) 2020 S. Karger AG, Basel
(C) 2020 S. Karger AG, Basel

www.karger.com/oc

Karger"

\section{Introduction}

Cholangiocarcinoma (CCA) is a malignant neoplasm arising from the biliary epithelium and peribiliary glands [1], representing $10 \%$ of hepatobiliary tumors and $2 \%$ of malignant tumors $[2,3]$. Traditionally, CCA is divided into three types based on anatomic location or growth pattern, including intrahepatic cholangiocarcinoma (ICC), perihilar cholangiocarcinoma, and distal cholangiocarcinoma [4]. The prognosis of CCA is very poor. The current 5-year survival rate after surgery rarely exceeds $35 \%$. Despite the relative rarity of CCA, the incidence and mortality rates of the disease have been reported to be gradually increasing worldwide, and it is the chief cause of nearly $20 \%$ of the deaths from hepatobiliary cancers $[5,6]$. Epidemiological investigations have indicated that men have a 1.5-fold increased risk of CCA development when compared with women [7].

The only effective way to cure CCA is complete surgical resection, which is only appropriate for patients with well-localized lesions. However, late presentation in many cases results in vascular encasement with lymphatic and perineural invasion, and curative surgical resection 
with negative tumor margins can only be offered to less than $30 \%$ of patients [8]. Furthermore, $20-50 \%$ of patients considered resectable based on preoperative workup are found to have unresectable disease during exploration $[9,10]$. Therefore, it is critical to accurately evaluate CCA for optimal treatment planning and for determining prognosis.

To date, multiple imaging techniques have been utilized for preoperative evaluation of CCA, including ultrasonography (US) [11-13], computerized tomography (CT) [14-16], positron emission tomography (PET) [17, 18], magnetic resonance imaging (MRI) [19-21], and cholangioscopy [22, 23]. Meanwhile, artificial intelligence (AI) techniques have influenced changes across healthcare and have been used in many biomedical areas, especially oncology [24]. Now, the application of AI, allowing machines to better represent and interpret complex data, has been increasing gradually in CCA evaluation [25-28].

\section{Preoperative Imaging Evaluation of CCA}

Although the gold standard of diagnosis, grading, and staging for CCA is still pathological examination, it is an invasive method and not appropriate for all patients with CCA. Recent studies indicate that imaging methods can also provide an accurate preoperative evaluation of CCA, resulting in better treatment selection and improved prognosis.

\section{Ultrasonography Technique}

Ultrasonography (US) is frequently the initial imaging modality performed to evaluate patients who are suspected to have biliary obstruction or a liver mass, in real time and noninvasively. Early studies on patients with obstructive jaundice praised the ability of US to identify bile duct dilatation and deplored its inability to accurately identify the pathogenesis of biliary obstruction. However, the results of some later studies, which were carried out with modern high-resolution US equipment, have countered these initial impressions. The sensitivity and specificity of US in the detection of perihilar cholangiocarcinoma have risen over the past 15 years, from a reported low of $33 \%$ in 1983 to a reported high of $96 \%$ in 1996 [29, 30]. It may allow avoiding more invasive procedures in some patients and help identify those patients for whom further investigation combined with modern color Doppler imaging, contrast-enhanced US, and so on might be useful.

\section{Contrast-Enhanced US}

Contrast-enhanced US has been reported to be used in preoperative evaluation of CCA - for example, the vascular pattern of the tumor, differential diagnosis with hepatic inflammatory lesions, and correlation with clinicopathologic findings and prognosis - indicating great potential for the evaluation of both luminal and extraluminal masses in CCA diagnosis [13, 31, 32].

\section{Intraductal US}

Intraductal US (IDUS) is a valuable, albeit uncommonly used, imaging modality which can be easily performed during endoscopic retrograde cholangiopancreatography (ERCP) and is suitable for visualizing narrow ductal cavities such as the bile duct and pancreatic duct [33]. In recent years, some studies have indicated that IDUS has a significant role in distinguishing malignant bile duct obstructions from benign ones with a rate of accuracy of around $90 \%[33,34]$.

\section{Endoscopic US}

Currently, endoscopic US (EUS) is recognized as an important tool for diagnosing pancreaticobiliary disease, which is helpful to display the morphology of a bile duct stricture clearly (such as any irregularity and its wall thickness), to stage regional lymph node and portal vein involvement and to facilitate diagnostic tissue acquisition [11]. An early study indicated that EUS had the same sensitivity and specificity in differentiating bile duct obstruction combined with ERCP, magnetic resonance cholangiopancreatography (MRCP), and CT [35]. EUS-guided fine needle aspiration (EUS-FNA) may establish the diagnosis of bile duct obstruction with a sensitivity for the diagnosis of malignancy ranging from 27 to $83 \%$, indicating the potential value of preoperative evaluation for CCA [36].

It should be noted that gastrointestinal gas and obesity frequently obscure the distal bile duct, and the result of transabdominal US is significantly influenced by the experience of the ultrasonographer and the quality of the equipment. In addition, IDUS requires the addition of ERCP at the same sitting, and has inferior performance for staging regional adenopathy and evaluation after biliary stent placement [37]. Importantly, tissue acquisition with EUS-FNA from suspected malignant proximal biliary strictures has been reported to have the potential risk of tumor seeding along the needle track [36]. Besides, EUS-FNA is also operator dependent and difficult to perform widely. 


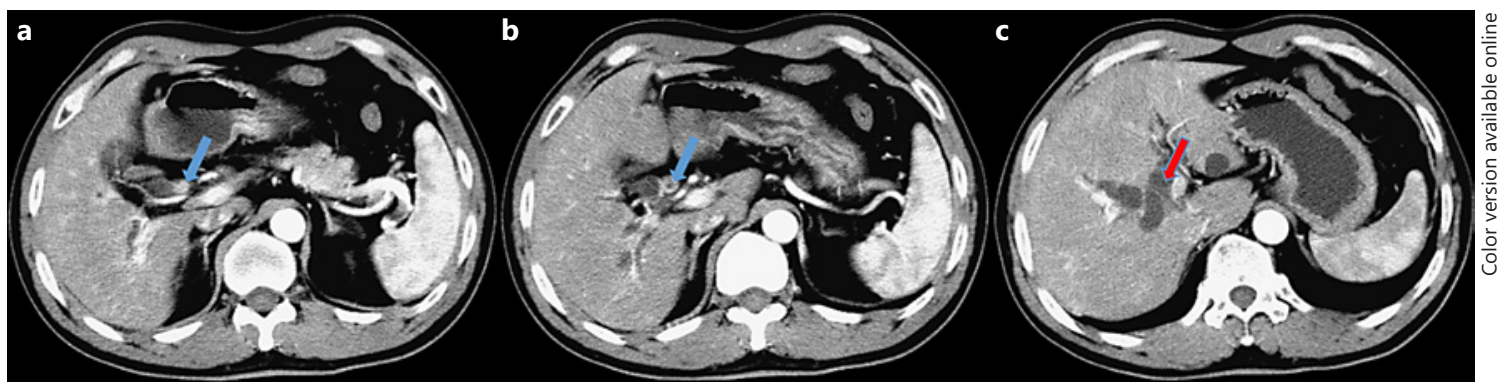

Fig. 1. Contrast-enhanced computerized tomography images of a 50-year-old male with infiltrative distal common bile duct (CBD) cholangiocarcinoma. The images (arterial phase) show the annular thickening and obvious enhancement of the wall of the distal CBD (a, b, blue arrows), with the intrahepatic and extrahepatic bile ducts dilated (c, red arrow).

\section{Computerized Tomography}

Multi-detector CT, which is noninvasive and has high spatial resolution in multiple imaging planes, is widely available to detect hepatobiliary disease. Multi-detector $\mathrm{CT}$, especially contrast-enhanced CT, has been commonly used for the characterization of liver masses; the detection of bile (Fig. 1) or pancreatic duct dilatation, stenosis, and vascular involvement; and the assessment of lymph nodes and distant metastases $[38,39]$. Meanwhile, the use of multiple postprocessing techniques, such as multi-planar reconstruction, curved planar reformation, maximum intensity projection, and 3D volume rendered images, is critical to evaluate the craniocaudal extent of infiltrative or intraluminal polypoidal lesions [40].

Recent research has demonstrated that CT techniques can effectively differentiate between CCA and other diseases, such as hepatocellular carcinoma (HCC) [41], liver abscess [42], and liver metastases [43]. It has been reported that CT perfusion or contrast-enhanced CT may assist in the diagnosis and differential diagnosis of CCA, which could guide optimal clinical treatment strategies [44-46]. In addition, recent studies have shown that dual-energy CT was also used to evaluate CCA and for the differentiation between small ICC and small liver abscess, which provided a new method of quantitatively assessing CCA $[47,48]$.

CT techniques are also able to accurately calculate liver volume and residual liver volume, assisting in making an optimal individualized surgical plan, leading to shorter surgical durations and less intraoperative blood loss, improving the surgical success rate, and reducing the incidence of operative complications [49]. Furthermore, these techniques can help to retain as much of the liver volume as possible and to reduce the risk of postoperative liver failure [49]. Survival outcomes in surgical patients can also be identified based on the qualitative imaging features of CCA on CT, resulting in a better prediction of prognosis [16].

However, some shortcomings in this modality are noteworthy. CT has ionizing radiation and may be harmful to overall patient health. Although CT can detect the dilation and stricture of bile ducts with a high degree of sensitivity, it has a weaker ability to evaluate the spread of the tumor along bile ducts and has a lower sensitivity for the preoperative evaluation of lymph node involvement than MRI [50].

\section{PET Imaging}

Positron Emission Tomography/Computerized

Tomography

Fluorine-18 fluorodeoxyglucose PET CT $\left({ }^{18} \mathrm{~F}-\mathrm{FDG}\right.$ $\mathrm{PET} / \mathrm{CT}$ ), a noninvasive imaging technique that allows in vivo assessment of the metabolic processes underlying malignant disease [51], is widely used in oncology imaging to diagnose and monitor the treatment response of cancer. To date, ${ }^{18} \mathrm{~F}$-FDG PET/CT has been widely used to evaluate multiple malignancies such as colorectal cancer [52], lung cancer [53], and breast cancer [54]. Various studies have indicated that ${ }^{18} \mathrm{~F}$-FDG PET/CT plays an important role in assessing CCA and have estimated the efficiency to detect and diagnose CCA at sensitivities of 84$94 \%$ and specificities of $79.3-100 \%[55,56]$. Also, PET/ CT has the advantage of the detection of lymph node metastases (LNM) and distant metastases of CCA [17], with a sensitivity up to $100 \%$ in detecting distant metastases [57]. Meanwhile, PET/CT also excels at differentiating ICC from distal cholangiocarcinoma based on metabolic behavior, especially FDG uptake, having an advantage in predicting survival and prognosis [58]. 


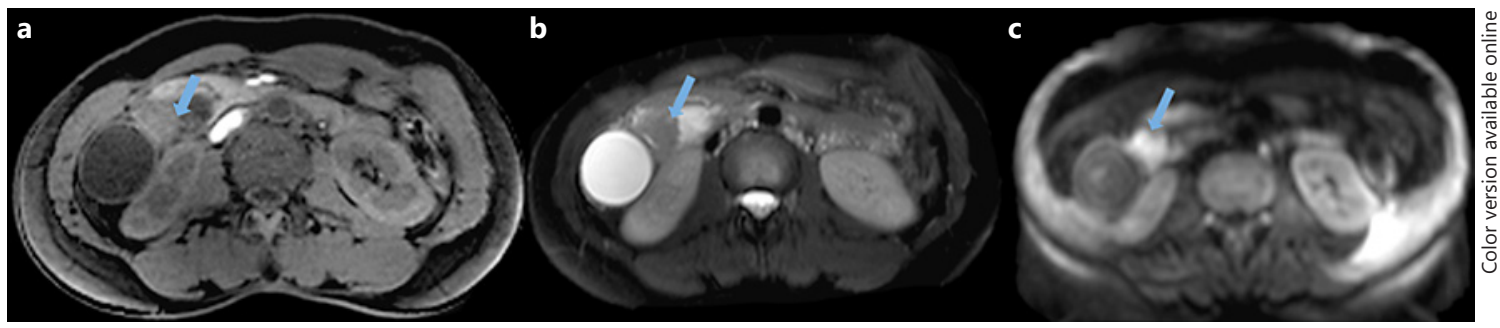

Fig. 2. Images of a 43-year-old female with distal common bile duct cholangiocarcinoma (blue arrows). Axial T1-weighted imaging (a) and fat-suppressed T2-weighted imaging (b) demonstrate an intermediate signal of a soft-tissue nodule, with a high signal on axial diffusion-weighted imaging (c).

Of course, other tracers such as (11)C-choline and ${ }^{68} \mathrm{Ga}$-prostate-specific membrane antigen have been also proven to be a promising method for assessing CCA, which may assist in the detection and finding of therapeutic options for CCA $[59,60]$.

However, PET/CT also has some limitations. First, it has a high cost and is not suitable for routine clinical examination. Second, PET alone is limited by poor temporal and spatial resolution with a somewhat restricted anatomic localization of positive lesions [40]. Finally, other limitations include misinterpretation of normal physiological activity of the bowel and genitourinary system and misregistration [40]. False-negative findings and falsepositive results are possible with some lesions, leading to diagnostic obscurity $[40,61]$.

Positron Emission Tomography/Magnetic Resonance Imaging

In recent studies, ${ }^{18} \mathrm{~F}$-FDG PET/MRI has been attempted for discriminating the histopathologic subtypes and histological grades of hepatic tumors, which may help to differentiate CCA from other hepatic neoplasms and predict the differentiation degree (DD) of CCA [62, 63]. Besides, Ferrone et al. [64] investigated management implications of PET/MRI in patients with untreated ICC, discovering that PET/MRI significantly influenced the treatment strategy in part of patients with ICC. Two quantitative parameters, including the maximum standard uptake value and apparent diffusion coefficient, were obtained simultaneously to provide complementary information on hepatic tumors by using PET/MRI scanning, indicating that PET/MRI may be a useful tool for the diagnosis, as well as for gauging the aggressiveness and prognosis, of a tumor, including CCA.

Regrettably, like PET/CT, PET/MRI has still a high cost and shows some similar disadvantages. Moreover,

Cholangiocarcinoma Evaluation via

Imaging and Artificial Intelligence
PET/MRI has longer scan times and more contraindications than PET/CT.

\section{Magnetic Resonance Imaging}

At present, MRI is considered the most accurate and least invasive imaging modality for the assessment of CCA (Fig. 2) [65]. MRI has the additional advantages of using no ionizing radiation, offering a high-quality softtissue contrast and tumor evaluation, as well as precise visualization of bile ducts and adjacent structures. These improvements are achieved through the combination of multiple sequences, including T1-weighted imaging [66], T2-weighted imaging [67], diffusion-weighted imaging (DWI) [21], MRCP [19], and dynamic contrast-enhanced MRI (DCE-MRI) [20, 65]. MRI techniques excel at visualizing malignant stricture with or without an associated mass, permit accurate lesion characterization, improve sensitivity in the evaluation of tumor extent along the bile duct and liver invasion, and provide staging information regarding vascular, ductal and lymph node status [68].

Although the ability of MRCP to differentiate the causes of structural disease is limited, and cannot alone be used in assessing resectability in most situations, it has been proven to be an optimal imaging modality for the evaluation of the biliary system, particularly excelling at assessing the tumor extent along the bile ducts [68] and visualizing the biliary ducts proximal to an obstruction [65]. Previous studies have documented that MRCP can differentiate benign from malignant causes of biliary obstruction [19] and assess the possibility of resectability [69].

It has been proven that DWI can increase the conspicuity of lesions, improving the diagnostic sensitivity of MRI for CCA [70]. It may also contribute to the differentiation between ICC (Fig. 3) and other hepatic malignancies, such as solitary hypovascular liver metastases [71] 


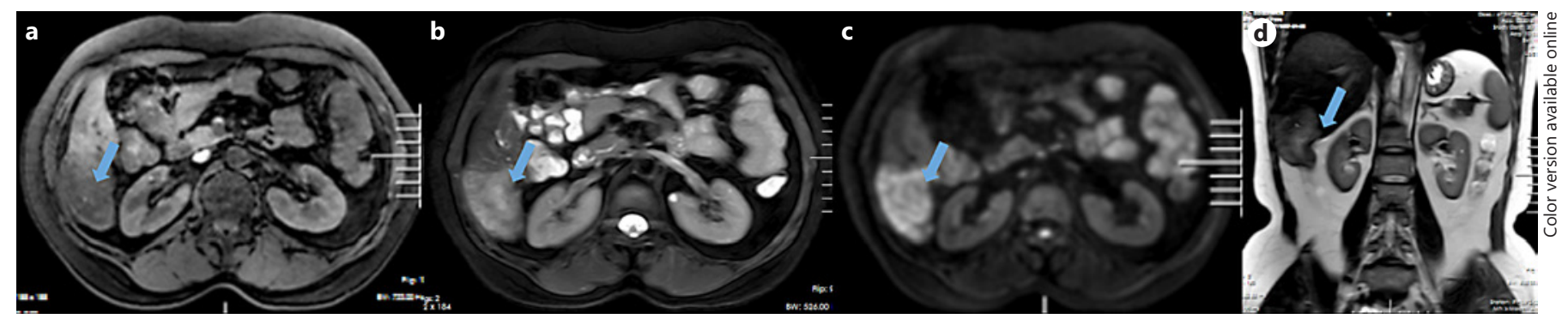

Fig. 3. Images of a 54-year-old male with a segment VI intrahepatic cholangiocarcinoma (blue arrows). The lesion has a low signal on axial T1-weighted imaging (a), and a heterogeneous high signal on axial fat-suppressed T2-weighted imaging (T2WI) (b), axial diffusion-weighted imaging (c), and coronary non-fat-suppressed T2WI (d).

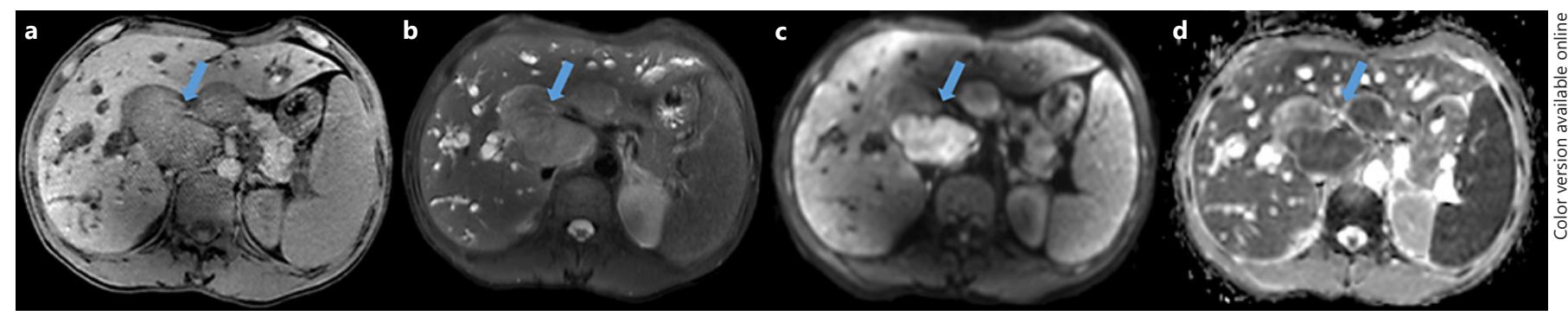

Fig. 4. Images of a 38-year-old man with a perihilar cholangiocarcinoma (blue arrows) invading the caudate lobe with intrahepatic bile duct dilatation. The tumor shows a slightly lower signal on axial T1-weighted imaging (a), an intermediate signal on axial fat-suppressed T2-weighted imaging (b), a high signal on axial diffusion-weighted imaging (c), and a low signal on the axial apparent diffusion coefficient $(\mathbf{d})$.

and HCC (Fig. 4) [72]. Furthermore, DWI may serve as an independent tool to evaluate histopathologic findings and the prognosis of patients with CCA $[73,74]$. It also allows a more accurate assessment of tumor status and the presence of associated satellite lesions when combined with DCE-MRI [75]. DCE-MRI may assist with a more accurate discrimination of intrahepatic mass-forming CCA from HCC [76] and improve prediction of the postoperative prognosis of intrahepatic mass-forming CCA [20].

Besides, intravoxel incoherent motion (IVIM) combined with DWI has been used to differentiate ICC from HCC, with the highest area under curve (AUC) of 0.803 , suggesting that IVIM and DWI parameters can be useful in differentiating ICC from HCC and may be helpful in choosing a treatment plan and predicting prognosis $[77$, 78]. In another study, researchers predicted Ki-67 expression in CCA through IVIM and DWI (highest AUC = 0.880 ), which could reflect the proliferative activity of CCA and predict the degree of malignancy of the tumor to some extent [79].
One of the limitations to MRI is a long scanning time, which requires little to no patient movement. Many patients have difficulty remaining still enough to secure highest-quality images. In addition, some patients may not be suited for MRI due to contraindications, including patients with severe claustrophobia, intracranial aneurysm clips, cardiac pacemakers, cochlear implants, and other metals in their bodies [40].

\section{Direct Cholangiography}

Direct cholangioscopy, considered the standard of reference for evaluating the ductal extent of a tumor, allows the direct visualization of the biliary system and may help in the differentiation of unclear bile duct strictures [80]. ERCP and percutaneous transhepatic cholangiography are often required for the diagnosis and management of CCA, as tissue sampling and cytological and/or histological confirmation of the diagnosis are achievable by washing, brushing, or intraductal biopsy [23]. Cholangioscopic targeted biopsies of biliary lesions appear to improve the diagnostic yield and show accuracy rates of up to $90 \%$ 


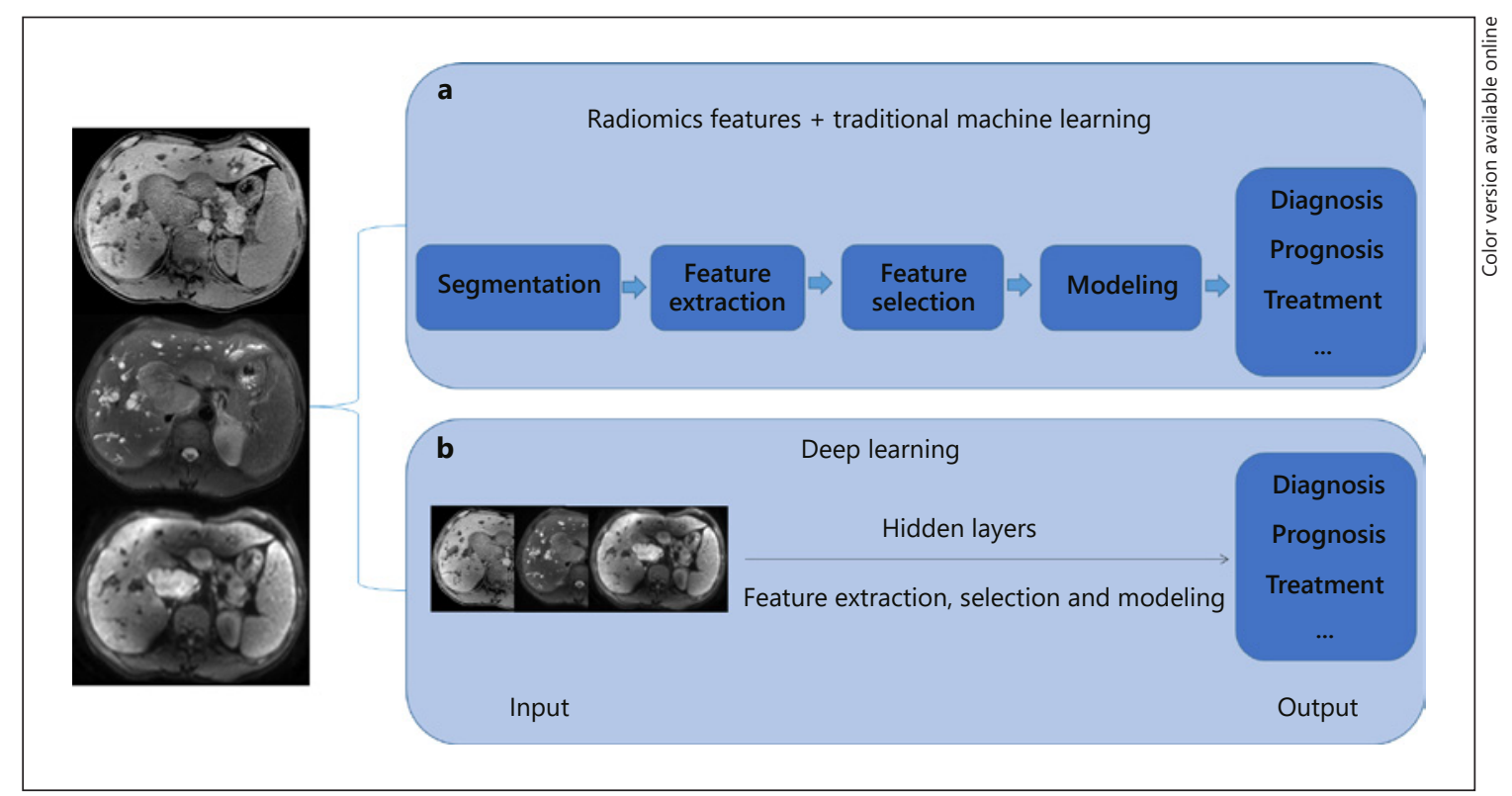

Fig. 5. Artificial intelligence methods in medical imaging. a Traditional flowchart of radiomics analysis, including segmentation of regions of interest (ROI), feature extraction, feature selection, and modeling. It relies on radiomics features extracted from ROI and conventional machine learning algorithms. b A new deep learning

in detecting CCA [22]. More importantly, therapeutic drainage, as a valuable treatment modality for patients with CCA, also can be undertaken by using plastic or metal stents [80].

Both ERCP and percutaneous transhepatic cholangiography, however, are invasive, operator dependent, and associated with procedural risks, including duodenal perforation, biliary leakage, cholangitis, pancreatitis, and bleeding [80]. Besides, the sensitivity of these approaches remains limited. It has been reported that the sensitivity of transpapillary forceps biopsies in detecting malignant bile duct strictures ranges only from 43 to $81 \%$ [81]. In perihilar biliary obstruction, direct cholangiography frequently does not depict the ductal anatomic features proximal to occlusive lesions, especially in cases of highgrade obstruction [80].

\section{Al in Medical Imaging}

In recent years, AI techniques have resulted in many improvements across healthcare and have been used in many biomedical areas, especially focused on oncology (Fig. 5). AI, which plays an important role in the medical field, can be used for drug discovery, remote patient mon-

Cholangiocarcinoma Evaluation via

Imaging and Artificial Intelligence algorithm, which also falls under radiomics but does not require region annotation. It consists of some hidden layers where radiomics feature extraction, selection, and ultimate modeling are performed simultaneously during training.

itoring, medical diagnostics and imaging, risk management, virtual assistance, and hospital management [24]. The major devices of AI consist of machine learning techniques and natural language processing methods [82]. Currently, large amounts of imaging data, coupled with data on clinical outcomes, have led to the emergence of AI within radiology and rapid development of radiomics as a new field of medical research [83].

Radiomics consists of the quantitative analysis of radiological images and machine learning methods [83]. The process extracts quantitative image features, also called "radiomics features," to achieve richer information about the intensity, shape, size, volume, and texture of tumor phenotypes that are distinct from or complementary to information provided by clinical reports, laboratory test results, and genomic or proteomic assays [84]. Diagnostic, prognostic, and predictive models are then built through the integration of features extracted from large-scale radiological image modalities (e.g., MRI, CT, PET, and US) to support personalized clinical decisions and improve individualized treatment selection [85].

Recently, radiomics tools have been successfully explored to assist clinical decision-making related to the diagnosis and risk stratification of different cancers. For example, studies in gliomas have used radiomics to predict 
the grading, molecular subtyping, and isocitrate dehydrogenase genotype, as well as low-grade glioma-related epilepsy, response to treatment, and the overall survival rate [86-89]. In addition, there are many related radiomics studies on non-small cell lung cancer that could predict distant metastasis in lung adenocarcinoma [90], histological tumor subtypes [91], disease recurrence [92], somatic mutations [93], gene expression profiles [94], and the overall survival rate [95]. Moreover, radiomics tools have also been applied to explore and evaluate other oncological foci, such as the prediction of immunohistochemical molecular classification in breast cancers [96], the differentiation of five liver masses [97], the prediction of complete response after neoadjuvant chemoradiation for locally advanced rectal cancer [98], and the preoperative prediction of LNM in bladder cancer [99]. It has been reported that radiomics approaches have also been applied to a number of non-oncological fields. The change in texture feature values between pre- and post-radiotherapy CT scans could be used to characterize radiationinduced damage to the lung [100]. Possible neurological applications include the diagnosis, staging, and prognosis of Alzheimer's and Parkinson's disease [101], as well as of multiple sclerosis [102]. In addition, the liver fibrosis stage could also be evaluated using a deep convolutional neural network [103]. The emerging field of radiomics has considerable potential in disease diagnosis, prognostic evaluation, and prediction of treatment response. AI can use sophisticated algorithms to extract and analyze features from a large volume of clinical and radiological data, and then use the obtained insights to guide clinical practice [82], assist in early detection and diagnosis, and make real-time inferences for health risk alerts and health outcome prediction of some diseases [83].

\section{Application of Al in CCA}

Although the role of AI in the evaluation of patients with CCA is not as established as routine imaging detection, $\mathrm{AI}$ is increasingly used in detecting and diagnosing CCA.

A previous paper demonstrated that an artificial neural network (ANN) was designed to differentiate four hepatic masses (HCC, intrahepatic peripheral CCA, hemangioma, and metastasis), reporting that the average AUC for ANN alone was 0.961. The ANN had the capability to differentiate some hepatic masses by use of CT and could improve the diagnostic accuracy of radiologists [104]. In another study, a popular ANN, the multi-layer perceptron, was used for differentiating images with CCA from those without. As a result, the achieved test was $94 \%$ correct when differentiating only healthy from tumor images, and $88 \%$ correct in a robust multi-disease test, indicating that using a multi-layer perceptron can accurately detect CCA in 2D MRCP images [25]. Pattanapairoj et al. [26] constructed a classification model using both C4.5 (which is an algorithm used to construct a decision tree classification model in a logical form) and an ANN to improve the discriminatory ability of certain serum markers for the diagnosis of CCA.

In a recent retrospective study, researchers discovered that certain texture parameters correlate significantly with epidermal growth factor receptor and vascular endothelial growth factor expression levels, suggesting that radiogenomic methods may predict protein expression of CCA and have a potential impact on therapy [27]. In addition, a novel radiomics nomogram based on radiomics signatures and clinical characteristics can be used to preoperatively predict early recurrence of ICC after partial hepatectomy, resulting in devising appropriate strategies, which benefits patients with a high risk of early recurrence who need additional chemotherapy [28]. This result is best shown in a study, comprising a total of 288 patients, in which Shao et al. [105] constructed an ANN model to accurately predict early occlusion of bilateral plastic stent placement for inoperable HCC, which is critical in treatment selection for patients with inoperable CCA. In a recent study, Ji et al. [106] established and validated a radiomics model for predicting LNM of ICC and determined its prognostic value, with good calibration and discrimination in the primary cohort $(\mathrm{AUC}=0.8462)$ and the validation cohort $(A U C=0.8921)$. Similarly, a radiomics model derived from portal-phase CT of the liver was developed to predict LNM and survival outcomes in biliary tract cancer, with an AUC of 0.81 and 0.80 in the primary cohort and the validation cohort, respectively. In other words, a noninvasive and convenient radiomics model that incorporates the radiomics signature and CT-reported lymph node status may facilitate clinical decision-making and potentially improve survival outcomes in selected patients [107].

In a recent study, a radiomics approach based on a support vector machine (SVM) using MRI was used to preoperatively evaluate LNM in ICC. The combination nomogram based on the SVM score, the CA19-9 level, and the MR-reported LNM factor showed better discrimination than the SVM model alone (AUC for the training group: 0.842 vs. 0.788 ; AUC for the validation group: 0.870 vs. 0.787 ) [108]. Another study consisted of
78

Oncology 2021;99:72-83

DOI: $10.1159 / 000507449$
Yang/Shu 
Table 1. Different applications of AI in CCA

\begin{tabular}{|c|c|c|c|c|c|c|}
\hline First author [Ref.] & Year & Images & Methods & $\begin{array}{l}\text { Subj } \\
n\end{array}$ & Purpose & AUC \\
\hline Matake et al. [104] & 2006 & CECT & ANN & 120 & $\begin{array}{l}\text { To evaluate the performance of ANN for differential diagnosis of hepatic } \\
\text { masses, including CCA }\end{array}$ & 0.961 \\
\hline Logeswaran [25] & 2009 & MRCP & MLP & 648 & To differentiate images with CCA from those without & - \\
\hline Pattanapairoj et al. [26] & 2015 & No & C4.5, ANN & 85 & To improve the diagnostic power of serum markers using C4.5 and ANN & - \\
\hline Sadot et al. [27] & 2015 & CECT & $\begin{array}{l}\text { Multiple linear } \\
\text { regression } \\
\text { analysis }\end{array}$ & 56 & $\begin{array}{l}\text { To investigate associations between imaging features of CCA and texture } \\
\text { analysis }\end{array}$ & - \\
\hline Liang et al. [28] & 2018 & CECT & LASSO & 209 & To develop a novel radiomics nomogram for predicting ER of ICC & 0.90 \\
\hline Shao et al. [105] & 2018 & No & BP-ANN & 288 & $\begin{array}{l}\text { To predict early occlusion of bilateral plastic stent placement for inoperable } \\
\text { HCC }\end{array}$ & 0.9648 \\
\hline Ji et al. [106] & 2019 & CECT & LASSO & 103 & $\begin{array}{l}\text { To develop a radiomics model for predicting LNM of ICC and to determine } \\
\text { its prognostic value }\end{array}$ & 0.9244 \\
\hline Ji et al. [107] & 2019 & CECT & LASSO & 247 & $\begin{array}{l}\text { To evaluate a radiomics model for predicting LNM in BTCs and to } \\
\text { determine its prognostic value }\end{array}$ & 0.81 \\
\hline Xu et al. [108] & 2019 & MRI & SVM & 148 & To develop a prediction model for preoperative LNM in ICC patients & 0.870 \\
\hline Peng et al. [109] & 2019 & US & LASSO, SVM & 128 & $\begin{array}{l}\text { To develop radiomics signatures based on US to assess the biological } \\
\text { behaviors of ICC }\end{array}$ & 0.930 \\
\hline Yang et al. [110] & 2020 & MRI & Random forest & 100 & $\begin{array}{l}\text { To evaluate diagnostic performance of radiomics models of MRI in the } \\
\text { detection of DD and LNM of ECC }\end{array}$ & 0.90 \\
\hline
\end{tabular}

The AUC values in the table were the best results in the above studies. AI, artificial intelligence; AUC, area under the curve; ANN, artificial neural network; CECT, contrast-enhanced computerized tomography; CCA, cholangiocarcinoma; MRCP, magnetic resonance cholangiopancreatography; MLP, multi-layer perceptron; C4.5, an algorithm used to construct a decision tree classification model in a logical form; ER, early recurrence; ICC, intrahepatic cholangiocarcinoma; LASSO, least absolute shrinkage and selection operator; BP-ANN, back-propagation artificial neural network; HCC, hilar cholangiocarcinoma; LNM, lymph node metastasis; BTCs, biliary tract cancers; SVM, support vector machine; MRI, magnetic resonance imaging; US, ultrasound; DD, differentiation degree; ECC, extrahepatic cholangiocarcinoma.

128 ICC patients' established and validated radiomics signatures based on US images to assess the biological behavior of ICC in a noninvasive manner, providing a novel approach to precision medicine for ICC patients [109]. Furthermore, in our previous study, we evaluated the diagnostic performance of radiomics models of MRI in the detection of DD and LNM of extrahepatic cholangiocarcinoma (ECC) through random forests, discovering that the radiomics models showed better performance in both the training and the testing cohort in predicting DD and LNM of ECC, with the highest AUC reaching 0.90 . The above results suggest that the radiomics models based on MRI performed well in predicting DD and LNM of ECC and have significant potential in clinical noninvasive diagnosis and in the prediction of ECC [110] (Table 1).

As we all know, the diagnosis or differential diagnosis of CCA or other tumors is sometimes difficult and requires much experience and knowledge because of the variety in radiological features of each disease and the overlap of radiological findings between many diseases [105]. Within the present detection workflow, radiologists are usually trained to identify abnormalities on the basis of changes in imaging intensities or the appearance of unusual patterns, relying on subjective perceptive and cognitive skills [24]. However, in contrast to such qualitative evaluation, AI can recognize complex patterns in imaging data and provide a quantitative and objective assessment of tumors automatically. Moreover, a more accurate and reproducible radiological assessment can be achieved with the integration of AI into the clinical workflow [24]. Therefore, we can evaluate CCA more accurately by combining AI with imaging to determine clinical management and prognostic evaluation. 


\section{Conclusions}

CCA is still a malignant neoplasm with an extremely unfavorable prognosis. Recent advances in imaging techniques have led to improved detection, characterization, and pre-treatment staging of these lesions, which in turn guides clinicians to make optimal therapeutic strategies. Our vision for the application of AI in CCA is expansive and bold. We expect that AI analysis is not only restricted to retrospective, single-center, and small-sample studies. More significantly, prospective multicenter studies with large data sets are needed to further predict CCA, including pathology, prognosis, treatment, and so on. Meanwhile, some clinical, pathological, radiological, and even genomic features should be considered in the analysis of AI for CCA besides for images.

For the immediate future, AI, especially in the field of imaging, will focus on the creation of suitable infrastructures to facilitate the development and validation of models. We envision that AI, a novel technique, will facilitate the diagnosis, prognosis, and treatment of CCA, resulting in improved personalization and precision medicine. Moreover, picture archiving and radiomics knowledge systems of the future may identify, segment, and extract features from regions of interest, not only for evaluating CCA. In summary, AI is at its very early stages in CCA and many challenges need to be addressed. We believe that simultaneous and synergistic advances in imaging and AI will empower the next major breakthroughs in personalization and precision medicine.

\section{Acknowledgements}

The authors acknowledge the other members for their contributions to the research, including Lu Yang and Lu Lu Xu.

\section{Conflict of Interest Statement}

The authors of this manuscript declare no relationships with any companies whose products or services may be related to the subject matter of the article.

\section{Funding Sources}

This study is supported by the Health Committee of Sichuan province (19PJ151).

\section{Author Contributions}

Literature search and manuscript preparation: Chun Mei Yang; study concept and design and manuscript editing: Jian Shu.

\section{References}

1 Valls C, Ruiz S, Martinez L, Leiva D. Radiological diagnosis and staging of hilar cholangiocarcinoma. World J Gastrointest Oncol. 2013;5(7):115-26.

2 Nakeeb A, Pitt HA, Sohn TA, Coleman J, Abrams RA, Piantadosi S, et al. Cholangiocarcinoma. A spectrum of intrahepatic, perihilar, and distal tumors. Ann Surg. 1996;224(4): 463-5; discussion 73-5.

3 Deoliveira ML, Schulick RD, Nimura Y, Rosen C, Gores G, Neuhaus P, et al. New staging system and a registry for perihilar cholangiocarcinoma. Hepatology. 2011;53(4):136371.

$4 \mathrm{Hu} \mathrm{JH}$, Tang JH, Lin CH, Chu YY, Liu NJ. Preoperative staging of cholangiocarcinoma and biliary carcinoma using 18F-fluorodeoxyglucose positron emission tomography: a meta-analysis. J Investig Med. 2018;66(1):5261.

5 Everhart JE, Ruhl CE. Burden of digestive diseases in the United States part III: liver, biliary tract, and pancreas. Gastroenterology. 2009; 136(4):1134-44.

6 Razumilava N, Gores GJ. Surveillance for cholangiocarcinoma in patients with primary sclerosing cholangitis: effective and justified? Clin Liver Dis (Hoboken). 2016;8(2):43-7.
7 Moazzami B, Majidzadeh-A K, DooghaieMoghadam A, Eslami P, Razavi-Khorasani N, Iravani $\mathrm{S}$, et al. Cholangiocarcinoma: state of the art. J Gastrointest Cancer. 2020 [online ahead of print].

8 DeOliveira ML, Cunningham SC, Cameron JL, Kamangar F, Winter JM, Lillemoe KD, et al. Cholangiocarcinoma: thirty-one-year experience with 564 patients at a single institution. Ann Surg. 2007;245(5):755-62.

9 Rocha FG, Matsuo K, Blumgart LH, Jarnagin WR. Hilar cholangiocarcinoma: the memorial Sloan-Kettering Cancer Center experience. J Hepatobiliary Pancreat Sci. 2010; 17(4):490-6.

10 Nagino M, Ebata T, Yokoyama Y, Igami T, Sugawara G, Takahashi Y, et al. Evolution of surgical treatment for perihilar cholangiocarcinoma: a single-center 34-year review of 574 consecutive resections. Ann Surg. 2013; 258(1):129-40.

11 Navaneethan U, Njei B, Venkatesh PG, Lourdusamy V, Sanaka MR. Endoscopic ultrasound in the diagnosis of cholangiocarcinoma as the etiology of biliary strictures: a systematic review and meta-analysis. Gastroenterol Rep (Oxf). 2015;3(3):209-15.
12 Sotoudehmanesh R, Nejati N, Farsinejad M, Kolahdoozan S. Efficacy of endoscopic ultrasonography in evaluation of undetermined etiology of common bile duct dilatation on abdominal ultrasonography. Middle East J Dig Dis. 2016;8(4):267-72.

13 Yin S, Cui Q, Yan K, Yang W, Wu W, Bao L, et al. Effect of contrast-enhanced ultrasound on differential diagnosis of intrahepatic cholangiocarcinoma and arterial phase enhanced hepatic inflammatory lesions. Chin J Cancer Res. 2017;29(3):272-80.

14 Asayama Y, Nishie A, Ishigami K, Ushijima Y, Takayama Y, Okamoto D, et al. Prognostic significance of contrast-enhanced CT attenuation value in extrahepatic cholangiocarcinoma. Eur Radiol. 2017;27(6):2563-9.

15 Fujita N, Asayama Y, Nishie A, Ishigami K, Ushijima Y, Takayama Y, et al. Mass-forming intrahepatic cholangiocarcinoma: enhancement patterns in the arterial phase of dynamic hepatic CT - correlation with clinicopathological findings. Eur Radiol. 2017;27(2):498-506.

16 Aherne EA, Pak LM, Goldman DA, Gonen M, Jarnagin WR, Simpson AL, et al. Intrahepatic cholangiocarcinoma: can imaging phenotypes predict survival and tumor genetics? Abdom Radiol (NY). 2018;43(10):2665-72. 
17 Wang ZF, Lan XL, Xiao YR, Liu JP, Ji JS. Correlation between TNM staging of primary cholangiocarcinoma and the maximum standard uptake value of $18 \mathrm{~F}$-2-deoxy-D-glucose positron emission tomography with computerized tomography [in Chinese]. Zhonghua Yi Xue Za Zhi. 2017;97(39):3104-7.

18 Ma KW, Cheung TT, She WH, Chok KSH, Chan ACY, Dai WC, et al. Diagnostic and prognostic role of 18-FDG PET/CT in the management of resectable biliary tract cancer. World J Surg. 2018;42(3):823-34.

19 Suthar M, Purohit S, Bhargav V, Goyal P. Role of MRCP in differentiation of benign and malignant causes of biliary obstruction. J Clin Diagn Res. 2015;9(11):Tc08-12.

20 Kim S, An C, Han K, Kim MJ. Gadoxetic acid enhanced magnetic resonance imaging for prediction of the postoperative prognosis of intrahepatic mass-forming cholangiocarcinoma. Abdom Radiol (NY). 2019;44(1):11021.

21 Promsorn J, Soontrapa W, Somsap K, Chamadol N, Limpawattana $P$, Harisinghani M. Evaluation of the diagnostic performance of apparent diffusion coefficient (ADC) values on diffusion-weighted magnetic resonance imaging (DWI) in differentiating between benign and metastatic lymph nodes in cases of cholangiocarcinoma. Abdom Radiol (NY). 2019;44(2):473-81.

22 Kalaitzakis E, Webster GJ, Oppong KW, Kallis Y, Vlavianos P, Huggett M, et al. Diagnostic and therapeutic utility of single-operator peroral cholangioscopy for indeterminate biliary lesions and bile duct stones. Eur J Gastroenterol Hepatol. 2012;24(6):656-64.

23 Voigtländer T, Lankisch TO. Endoscopic diagnosis of cholangiocarcinoma: from endoscopic retrograde cholangiography to bile proteomics. Best Pract Res Clin Gastroenterol. 2015;29(2):267-75.

24 Hosny A, Parmar C, Quackenbush J, Schwartz $\mathrm{LH}$, Aerts HJWL. Artificial intelligence in radiology. Nat Rev Cancer. 2018;18(8):500-10.

25 Logeswaran R. Cholangiocarcinoma - an automated preliminary detection system using MLP. J Med Syst. 2009;33(6):413-21.

26 Pattanapairoj S, Silsirivanit A, Muisuk K, Seubwai W, Cha'on U, Vaeteewoottacharn K, et al. Improve discrimination power of serum markers for diagnosis of cholangiocarcinoma using data mining-based approach. Clin Biochem. 2015;48(10-11):668-73.

27 Sadot E, Simpson AL, Do RK, Gonen M, Shia J, Allen PJ, et al. Cholangiocarcinoma: correlation between molecular profiling and imaging phenotypes. PLoS One. 2015;10(7): e0132953.

28 Liang W, Xu L, Yang P, Zhang L, Wan D, Huang $Q$, et al. Novel nomogram for preoperative prediction of early recurrence in intrahepatic cholangiocarcinoma. Front Oncol. 2018;8:360
29 Honickman SP, Mueller PR, Wittenberg J, Simeone JF, Ferrucci JT, Cronan JJ, et al. Ultrasound in obstructive jaundice: prospective evaluation of site and cause. Radiology. 1983; 147(2):511-5

30 Robledo R, Muro A, Prieto ML. Extrahepatic bile duct carcinoma: US characteristics and accuracy in demonstration of tumors. Radiology. 1996;198(3):869-73.

31 Vilana R, Forner A, Bianchi L, García-Criado A, Rimola J, de Lope CR, et al. Intrahepatic peripheral cholangiocarcinoma in cirrhosis patients may display a vascular pattern similar to hepatocellular carcinoma on contrastenhanced ultrasound. Hepatology. 2010; 51(6):2020-9.

32 Yuan M, Li R, Zhang Y, Yang L, Zhang X, Tang C, et al. Enhancement patterns of intrahepatic cholangiocarcinoma on contrast-enhanced ultrasound: correlation with clinicopathologic findings and prognosis. Ultrasound Med Biol. 2019;45(1):26-34.

33 Chen L, Lu Y, Wu JC, Bie L, Xia L, Gong B. Diagnostic utility of endoscopic retrograde cholangiography/intraductal ultrasound (ERC/IDUS) in distinguishing malignant from benign bile duct obstruction. Dig Dis Sci. 2016;61(2):610-7.

34 Meister T, Heinzow HS, Woestmeyer C, Lenz P, Menzel J, Kucharzik T, et al. Intraductal ultrasound substantiates diagnostics of bile duct strictures of uncertain etiology. World J Gastroenterol. 2013;19(6):874-81.

35 Rösch T, Meining A, Fr ühmorgen S, Zillinger C, Schusdziarra V, Hellerhoff K, et al. A prospective comparison of the diagnostic accuracy of ERCP, MRCP, CT, and EUS in biliary strictures. Gastrointest Endosc. 2002;55(7): 870-6.

36 Levy MJ, Heimbach JK, Gores GJ. Endoscopic ultrasound staging of cholangiocarcinoma. Curr Opin Gastroenterol. 2012;28(3):244-52.

37 Levy MJ, Vazquez-Sequeiros E, Wiersema MJ. Evaluation of the pancreaticobiliary ductal systems by intraductal US. Gastrointest Endosc. 2002;55(3):397-408.

38 Baheti $A D$, Tirumani $S H$, Shinagare $A B$, Rosenthal MH, Hornick JL, Ramaiya NH, et al. Correlation of CT patterns of primary intrahepatic cholangiocarcinoma at the time of presentation with the metastatic spread and clinical outcomes: retrospective study of 92 patients. Abdom Imaging. 2014;39(6):1193-201.

39 Ciresa M, De Gaetano AM, Pompili M, Saviano $\mathrm{A}$, Infante $\mathrm{A}$, Montagna $\mathrm{M}$, et al. Enhancement patterns of intrahepatic massforming cholangiocarcinoma at multiphasic computed tomography and magnetic resonance imaging and correlation with clinicopathologic features. Eur Rev Med Pharmacol Sci. 2015;19(15):2786-97.

40 Ringe KI, Wacker F. Radiological diagnosis in cholangiocarcinoma: application of computed tomography, magnetic resonance imaging, and positron emission tomography. Best Pract Res Clin Gastroenterol. 2015;29(2): 253-65.
41 Sano S, Yamamoto Y, Sugiura T, Okamura Y, Ito $\mathrm{T}$, Ashida $\mathrm{R}$, et al. The radiological differentiation of hypervascular intrahepatic cholangiocarcinoma from hepatocellular carcinoma with a focus on the CT value on multiphase enhanced CT. Anticancer Res. 2018; 38(9):5505-12.

42 Joob B, Wiwanitkit V. Cholangiocarcinoma versus small liver abscess in dual source dualenergy CT quantitative parameters. Eur J Radiol. 2018;99:130.

43 Apisarnthanarak P, Pansri C, Maungsomboon $\mathrm{K}$, Thamtorawat $\mathrm{S}$. The $\mathrm{CT}$ appearances for differentiating of peripheral, mass-forming cholangiocarcinoma and liver meatastases [sic!] from colorectal adenocarcinoma. J Med Assoc Thai. 2014;97(4):415-22.

44 Inoue E, Fujita M, Hosomi N, Sawai Y, Hashimoto T, Kuroda C, et al. Double phase CT arteriography of the whole liver in the evaluation of hepatic tumors. J Comput Assist Tomogr. 1998;22(1):64-8.

45 Miura F, Okazumi S, Takayama W, Asano T, Makino H, Shuto K, et al. Evaluation of CT during arterial portography for preoperative diagnosis of intrahepatic cholangiocarcinoma. Hepatogastroenterology. 2006;53(69): 435-7.

46 D’Onofrio M, Vecchiato F, Cantisani V, Barbi E, Passamonti M, Ricci P, et al. Intrahepatic peripheral cholangiocarcinoma (IPCC): comparison between perfusion ultrasound and CT imaging. Radiol Med. 2008;113(1): 76-86.

47 Thomas JV, Bolus DN, Jackson BE, Berland LL, Yester M, Morgan DE. Gadoxetate disodium enhanced spectral dual-energy CT for evaluation of cholangiocarcinoma: preliminary data. Ann Med Surg (Lond). 2016;6:1722.

48 Kim JE, Kim HO, Bae K, Cho JM, Choi HC, Choi DS. Differentiation of small intrahepatic mass-forming cholangiocarcinoma from small liver abscess by dual source dual-energy CT quantitative parameters. Eur J Radiol. 2017;92:145-52.

49 Zeng N, Tao H, Fang C, Fan Y, Xiang N, Yang $\mathrm{J}$, et al. Individualized preoperative planning using three-dimensional modeling for Bismuth and Corlette type III hilar cholangiocarcinoma. World J Surg Oncol. 2016;14(1):44.

50 Nagino M. Perihilar cholangiocarcinoma: a surgeon's viewpoint on current topics. J Gastroenterol. 2012;47(11):1165-76.

51 Albazaz R, Patel CN, Chowdhury FU, Scarsbrook AF. Clinical impact of FDG PET-CT on management decisions for patients with primary biliary tumours. Insights Imaging. 2013; 4(5):691-700.

52 Grut H, Revheim ME, Line PD, Dueland S. Importance of 18F-FDG PET/CT to select patients with nonresectable colorectal liver metastases for liver transplantation. Nucl Med Commun. 2018;39(7):621-7.
Cholangiocarcinoma Evaluation via Imaging and Artificial Intelligence
Oncology 2021;99:72-83

DOI: $10.1159 / 000507449$ 
53 Pahk K, Chung JH, Yi E, Kim S, Lee SH. Metabolic tumor heterogeneity analysis by F-18 FDG PET/CT predicts mediastinal lymph node metastasis in non-small cell lung cancer patients with clinically suspected N2. Eur J Radiol. 2018;106:145-9.

54 Lei L, Wang X, Chen Z. PET/CT imaging for monitoring recurrence and evaluating response to treatment in breast cancer. Adv Clin Exp Med. 2016;25(2):377-82.

55 Jadvar H, Henderson RW, Conti PS. [F-18] fluorodeoxyglucose positron emission tomography and positron emission tomography: computed tomography in recurrent and metastatic cholangiocarcinoma. J Comput Assist Tomogr. 2007;31(2):223-8.

56 Kim JY, Kim MH, Lee TY, Hwang CY, Kim JS, Yun SC, et al. Clinical role of 18F-FDG PET-CT in suspected and potentially operable cholangiocarcinoma: a prospective study compared with conventional imaging. Am J Gastroenterol. 2008;103(5):1145-51

57 Petrowsky H, Wildbrett P, Husarik DB, Hany TF, Tam S, Jochum W, et al. Impact of integrated positron emission tomography and computed tomography on staging and management of gallbladder cancer and cholangiocarcinoma. J Hepatol. 2006;45(1):43-50.

58 Sabaté-Llobera A, Gràcia-Sánchez L, ReynésLlompart G, Ramos E, Lladó L, Robles J, et al. Differences on metabolic behavior between intra and extrahepatic cholangiocarcinomas at 18F-FDG-PET/CT: prognostic implication of metabolic parameters and tumor markers. Clin Transl Oncol. 2019;21(3):324-33.

59 Chotipanich C, Promteangtrong C, Kunawudhi A, Chanwat R, Sricharunrat T, Suratako S, et al. 11C-choline and FDG PET/CT imaging of primary cholangiocarcinoma: a comparative analysis. Asia Ocean J Nucl Med Biol. 2015;3(1):18-25.

60 Marafi F, Usmani S, Esmail A. 68Ga-prostatespecific membrane antigen PET/CT in cholangiocarcinoma: a potential biomarker for targeted radioligand therapy? Clin Nucl Med. 2019;44(7):e439-41.

61 Wakabayashi H, Akamoto S, Yachida S, Okano K, Izuishi K, Nishiyama Y, et al. Significance of fluorodeoxyglucose PET imaging in the diagnosis of malignancies in patients with biliary stricture. Eur J Surg Oncol. 2005; 31(10):1175-9.

62 Kong E, Chun KA, Cho IH. Quantitative assessment of simultaneous F-18 FDG PET/ MRI in patients with various types of hepatic tumors: correlation between glucose metabolism and apparent diffusion coefficient. PLoS One. 2017;12(7):e0180184.

63 Çelebi F, Yaghouti K, Cindil E, Dogusoy GB, Tokat Y, Balc1 C. The role of 18F-FDG PET/ MRI in the assessment of primary intrahepatic neoplasms. Acad Radiol. 2020 [online ahead of print].
64 Ferrone C, Goyal L, Qadan M, Gervais D, Sahani DV, Zhu AX, et al. Management implications of fluorodeoxyglucose positron emission tomography/magnetic resonance in untreated intrahepatic cholangiocarcinoma. Eur J Nucl Med Mol Imaging. 2019 [online ahead of print].

65 Jhaveri KS, Hosseini-Nik H. MRI of cholangiocarcinoma. J Magn Reson Imaging. 2015; 42(5):1165-79.

66 Sammon J, Fischer S, Menezes R, HosseiniNik H, Lewis S, Taouli B, et al. MRI features of combined hepatocellular-cholangiocarcinoma versus mass forming intrahepatic cholangiocarcinoma. Cancer Imaging. 2018; 18(1):8.

67 Hwang J, Kim YK, Min JH, Choi SY, Jeong WK, Hong SS, et al. Capsule, septum, and T2 hyperintense foci for differentiation between large hepatocellular carcinoma $(\geq 5 \mathrm{~cm})$ and intrahepatic cholangiocarcinoma on gadoxetic acid MRI. Eur Radiol. 2017;27(11):458190.

68 Fowler KJ, Saad NE, Linehan D. Imaging approach to hepatocellular carcinoma, cholangiocarcinoma, and metastatic colorectal cancer. Surg Oncol Clin N Am. 2015;24(1):1940.

69 Ayuso JR, Pagés M, Darnell A. Imaging bile duct tumors: staging. Abdom Imaging. 2013; 38(5):1071-81.

70 Fábrega-Foster K, Ghasabeh MA, Pawlik TM, Kamel IR. Multimodality imaging of intrahepatic cholangiocarcinoma. Hepatobiliary Surg Nutr. 2017;6(2):67-8.

71 Kovač JD, Galun D, Đurić-Stefanović A, Lilić G, Vasin D, Lazić L, et al. Intrahepatic massforming cholangiocarcinoma and solitary hypovascular liver metastases: is the differential diagnosis using diffusion-weighted MRI possible? Acta Radiol. 2017;58(12):1417-26.

72 Sheng RF, Zeng MS, Rao SX, Ji Y, Chen LL. MRI of small intrahepatic mass-forming cholangiocarcinoma and atypical small hepatocellular carcinoma $(\leq 3 \mathrm{~cm})$ with cirrhosis and chronic viral hepatitis: a comparative study. Clin Imaging. 2014;38(3):265-72.

73 Lee J, Kim SH, Kang TW, Song KD, Choi D, Jang KT. Mass-forming intrahepatic cholangiocarcinoma: diffusion-weighted imaging as a preoperative prognostic marker. Radiology. 2016;281(1):119-28.

74 Lewis S, Besa C, Wagner M, Jhaveri K, Kihira $\mathrm{S}$, Zhu H, et al. Prediction of the histopathologic findings of intrahepatic cholangiocarcinoma: qualitative and quantitative assessment of diffusion-weighted imaging. Eur Radiol. 2018;28(5):2047-57.

75 Feng ST, Wu L, Cai H, Chan T, Luo Y, Dong $Z$, et al. Cholangiocarcinoma: spectrum of appearances on Gd-EOB-DTPA-enhanced MR imaging and the effect of biliary function on signal intensity. BMC Cancer. 2015;15:38.
76 Kim R, Lee JM, Shin CI, Lee ES, Yoon JH, Joo I, et al. Differentiation of intrahepatic massforming cholangiocarcinoma from hepatocellular carcinoma on gadoxetic acid-enhanced liver MR imaging. Eur Radiol. 2016; 26(6):1808-17.

77 Wei Y, Gao F, Zheng D, Huang Z, Wang M, $\mathrm{Hu} \mathrm{F}$, et al. Intrahepatic cholangiocarcinoma in the setting of HBV-related cirrhosis: differentiation with hepatocellular carcinoma by using intravoxel incoherent motion diffusion-weighted MR imaging. Oncotarget. 2018;9(8):7975-83.

78 Shao S, Shan Q, Zheng N, Wang B, Wang J. Role of intravoxel incoherent motion in discriminating hepatitis B virus-related intrahepatic mass-forming cholangiocarcinoma from hepatocellular carcinoma based on Liver Imaging Reporting and Data System v2018. Cancer Biother Radiopharm. 2019;34(8):511-8.

79 Cui X, Chen H, Cai S, Tang Q, Fang X. Correlation of apparent diffusion coefficient and intravoxel incoherent motion imaging parameters with $\mathrm{Ki}-67$ expression in extrahepatic cholangiocarcinoma. Magn Reson Imaging. 2019;63:80-4.

80 Choi JY, Kim MJ, Lee JM, Kim KW, Lee JY, Han JK, et al. Hilar cholangiocarcinoma: role of preoperative imaging with sonography, MDCT, MRI, and direct cholangiography. AJR Am J Roentgenol. 2008;191(5):1448-57.

81 De Bellis M, Sherman S, Fogel EL, Cramer H, Chappo J, McHenry L, et al. Tissue sampling at ERCP in suspected malignant biliary strictures (Part 1). Gastrointest Endosc. 2002; 56(4):552-61.

82 Tekkeşin A. Artificial intelligence in healthcare: past, present and future. Anatol J Cardiol. 2019;22(Suppl 2):8-9.

83 Parekh V, Jacobs MA. Radiomics: a new application from established techniques. Expert Rev Precis Med Drug Dev. 2016;1(2):207-26.

84 Avanzo M, Stancanello J, El Naqa I. Beyond imaging: the promise of radiomics. Phys Med. 2017;38:122-39.

85 Gillies RJ, Kinahan PE, Hricak H. Radiomics: images are more than pictures, they are data. Radiology. 2016;278(2):563-77.

86 Liu Z, Wang Y, Liu X, Du Y, Tang Z, Wang K, et al. Radiomics analysis allows for precise prediction of epilepsy in patients with lowgrade gliomas. Neuroimage Clin. 2018;19: 271-8.

87 Lohmann P, Lerche C, Bauer EK, Steger J, Stoffels G, Blau T, et al. Predicting IDH genotype in gliomas using FET PET radiomics. Sci Rep. 2018;8(1):13328.

88 Lu CF, Hsu FT, Hsieh KL, Kao YJ, Cheng SJ, $\mathrm{Hsu} \mathrm{JB}$, et al. Machine learning-based radiomics for molecular subtyping of gliomas. Clin Cancer Res. 2018;24(18):4429-36.

89 Wang C, Sun W, Kirkpatrick J, Chang Z, Yin FF. Assessment of concurrent stereotactic radiosurgery and bevacizumab treatment of recurrent malignant gliomas using multi-modality MRI imaging and radiomics analysis. J Radiosurg SBRT. 2018;5(3):171-81. 
90 Coroller TP, Grossmann P, Hou Y, Rios Velazquez E, Leijenaar RT, Hermann G, et al. CT-based radiomic signature predicts distant metastasis in lung adenocarcinoma. Radiother Oncol. 2015;114(3):345-50.

91 Wu W, Parmar C, Grossmann P, Quackenbush J, Lambin P, Bussink J, et al. Exploratory study to identify radiomics classifiers for lung cancer histology. Front Oncol. 2016;6:71.

92 Huynh E, Coroller TP, Narayan V, Agrawal V, Romano J, Franco I, et al. Associations of radiomic data extracted from static and respiratory-gated $\mathrm{CT}$ scans with disease recurrence in lung cancer patients treated with SBRT. PLoS One. 2017;12(1):e0169172.

93 Rios Velazquez E, Parmar C, Liu Y, Coroller TP, Cruz G, Stringfield O, et al. Somatic mutations drive distinct imaging phenotypes in lung cancer. Cancer Res. 2017;77(14):392230.

94 Grossmann P, Stringfield O, El-Hachem N, Bui MM, Rios Velazquez E, Parmar C, et al. Defining the biological basis of radiomic phenotypes in lung cancer. Elife. 2017;6: e23421.

95 Huang Y, Liu Z, He L, Chen X, Pan D, Ma Z, et al. Radiomics signature: a potential biomarker for the prediction of disease-free survival in early-stage (I or II) non-small cell lung cancer. Radiology. 2016;281(3):947-57.

$96 \mathrm{Li} \mathrm{H}$, Zhu Y, Burnside ES, Huang E, Drukker $\mathrm{K}$, Hoadley KA, et al. Quantitative MRI radiomics in the prediction of molecular classifications of breast cancer subtypes in the TCGA/TCIA data set. NPJ Breast Cancer. 2016;2:16012.
97 Yasaka K, Akai H, Abe O, Kiryu S. Deep learning with convolutional neural network for differentiation of liver masses at dynamic contrast-enhanced CT: a preliminary study. Radiology. 2018;286(3):887-96.

98 Bibault JE, Giraud P, Housset M, Durdux C Taieb J, Berger A, et al. Deep learning and radiomics predict complete response after neo-adjuvant chemoradiation for locally advanced rectal cancer. Sci Rep. 2018;8(1): 12611.

99 Wu S, Zheng J, Li Y, Wu Z, Shi S, Huang M, et al. Development and validation of an MRI-based radiomics signature for the preoperative prediction of lymph node metastasis in bladder cancer. EBioMedicine. 2018; 34:76-84.

100 Cunliffe A, Armato SG, Castillo R, Pham N, Guerrero T, Al-Hallaq HA. Lung texture in serial thoracic computed tomography scans: correlation of radiomics-based features with radiation therapy dose and radiation pneumonitis development. Int J Radiat Oncol Biol Phys. 2015;91(5):1048-56.

101 Zhang J, Yu C, Jiang G, Liu W, Tong L. 3D texture analysis on MRI images of Alzheimer's disease. Brain Imaging Behav. 2012;6(1): 61-9.

102 Zhang J, Tong L, Wang L, Li N. Texture analysis of multiple sclerosis: a comparative study. Magn Reson Imaging. 2008;26(8): $1160-6$.

103 Yasaka K, Akai H, Kunimatsu A, Abe O, Kiryu S. Deep learning for staging liver fibrosis on CT: a pilot study. Eur Radiol. 2018; 28(11):4578-85.
104 Matake K, Yoshimitsu K, Kumazawa S, Higashida $\mathrm{Y}$, Irie $\mathrm{H}$, Asayama $\mathrm{Y}$, et al. Usefulness of artificial neural network for differential diagnosis of hepatic masses on CT images. Acad Radiol. 2006;13(8):951-62.

105 Shao F, Huang Q, Wang C, Qiu L, Hu YG, Zha SY. Artificial neural networking model for the prediction of early occlusion of bilateral plastic stent placement for inoperable hilar cholangiocarcinoma. Surg Laparosc Endosc Percutan Tech. 2018;28(2):e54-8.

106 Ji GW, Zhu FP, Zhang YD, Liu XS, Wu FY, Wang $\mathrm{K}$, et al. A radiomics approach to predict lymph node metastasis and clinical outcome of intrahepatic cholangiocarcinoma. Eur Radiol. 2019;29(7):3725-35.

107 Ji GW, Zhang YD, Zhang H, Zhu FP, Wang $\mathrm{K}$, Xia YX, et al. Biliary tract cancer at CT: a radiomics-based model to predict lymph node metastasis and survival outcomes. Radiology. 2019;290(1):90-8.

108 Xu L, Yang P, Liang W, Liu W, Wang W, Luo $\mathrm{C}$, et al. A radiomics approach based on support vector machine using MR images for preoperative lymph node status evaluation in intrahepatic cholangiocarcinoma. Theranostics. 2019;9(18):5374-85.

109 Peng YT, Zhou CY, Lin P, Wen DY, Wang $\mathrm{XD}$, Zhong XZ, et al. Preoperative ultrasound radiomics signatures for noninvasive evaluation of biological characteristics of intrahepatic cholangiocarcinoma. Acad Radiol. 2019 [online ahead of print].

110 Yang C, Huang M, Li S, Chen J, Yang Y, Qin $\mathrm{N}$, et al. Radiomics model of magnetic resonance imaging for predicting pathological grading and lymph node metastases of extrahepatic cholangiocarcinoma. Cancer Lett. 2020;470:1-7. 